\title{
Successful Conservative Management of Trimethoprim Induced Life-Threatening Hyperkalaemia in a Patient with Pneumocystis jirroveciil Pneumonia
}

\author{
Jamie Johnstone, Andrew Macduff \\ Department of Respiratory and Intensive Care Medicine, New Cross Hospital, Wolverhampton, UK \\ Email: jrjohnstone@doctors.org.uk
}

Received 30 June 2014; revised 25 July 2014; accepted 12 August 2014

Copyright (C) 2014 by authors and Scientific Research Publishing Inc.

This work is licensed under the Creative Commons Attribution International License (CC BY).

http://creativecommons.org/licenses/by/4.0/

\begin{abstract}
Co-trimoxazole is a combination antibiotic made up of trimethoprim and sulphamethoxazole that is first line treatment for Pneumocystis jirovecii pneumonia (PJP). Hyperkalaemia is a relatively common side effect of the trimethoprim component of co-trimoxazole but it is not well recognised by clinicians. The mechanism of action causing hyperkalaemia due to trimethoprim is similar to the potassium sparing diuretic effect of amiloride. It has been suggested on this basis that the hyperkalaemia can be reversed by the administration of furosemide and $0.9 \%$ saline to promote kaliuresis. We present what we believe to be the first published case of successfully managing trimethoprim induced hyperkalaemia with furosemide and $0.9 \%$ saline allowing the continued use of co-trimoxazole to treat severe PJP.
\end{abstract}

\section{Keywords}

Trimethoprim, Hyperkalaemia, Furosemide, Saline, PJP

\section{Introduction}

Pneumocystis jirovecii pneumonia (PJP) is a life-threatening illness that occurs in immunocompromised individuals. Co-trimoxazole is established as first-line therapy for PJP. Hyperkalaemia is a relatively common side effect of the trimethoprim component of co-trimoxazole, however, this is not widely appreciated by physicians. Hyperkalaemia is itself life-threatening and can require the initiation of aggressive therapies such as renal replacement.

How to cite this paper: Johnstone, J. and Macduff, A. (2014) Successful Conservative Management of Trimethoprim Induced Life-Threatening Hyperkalaemia in a Patient with Pneumocystis jirovecii Pneumonia. Case Reports in Clinical Medicine, 3, 469-473. http://dx.doi.org/10.4236/crcm.2014.38103 
By understanding the mechanism of how trimethoprim causes hyperkalaemia it appears possible to treat the hyperkalaemia conservatively with furosemide and $0.9 \%$ saline and continuing to use co-trimoxazole, the first line treatment for PJP. With the case below we demonstrate how trimethoprim causes hyperkalaemia, how it can be successfully treated in a conservative manor and remind our colleagues of this potentially fatal side effect.

\section{Case History}

A 62-year-old male presented with a history of dry cough, fever, myalgia and headache. He had a past medical history of retinal vasculitis and was immunosuppressed with $1500 \mathrm{mg}$ mycophenalate twice daily and $11 \mathrm{mg}$ prednisolone once daily. He had recently been treated as an in-patient for community acquired pneumonia. 2 days following discharge he was readmitted with respiratory distress. He was profoundly hypoxic with oxygen saturations of $40 \%$ on room air. On examination he had crepitations throughout both lung fields. An arterial blood gas taken on $15 \mathrm{~L} /$ minute oxygen showed pH 7.441, $\mathrm{pO}_{2} 7.14 \mathrm{kPa}, \mathrm{pCO}_{2} 3.20 \mathrm{kPa}, \mathrm{HCO}_{3} 18.6 \mathrm{mmol} / \mathrm{L}$, $\mathrm{BE}-6.9 \mathrm{mmol} / \mathrm{L}$ and lactate $1.3 \mathrm{mmol} / \mathrm{L}$. His CRP was $172 \mathrm{mg} / \mathrm{L}$, WCC $10.6 \times 10^{9} / \mathrm{L}$ and his chest $\mathrm{x}$-ray showed widespread pulmonary infiltrates (Figure 1). He was intubated in the emergency department and admitted to the Intensive Care Unit. He was commenced on methylprednisolone, piperacillin/tazobactam and clarithromycin. Given his immunosuppressed status he was also treated with intravenous co-trimoxazole for possible PJP infection while awaiting results of a bronchoalveolar lavage. Computed tomography (CT) of the thorax showed bilateral ground glass changes consistent with acute pneumonitis (Figure 2). Blood cultures, atypical pneumonia screen, cytomegalovirus (CMV) and human immunodeficiency virus (HIV) tests were all negative. He developed a right sided pneumothorax which was treated by insertion of an intercostal drain.

On day 4 of his admission his potassium rose to $6 \mathrm{mmol} / \mathrm{L}$. At this point his renal function was acceptable with a creatinine of $112 \mu \mathrm{mol} / \mathrm{L}$, he had a good urine output (over $0.5 \mathrm{ml} / \mathrm{kg} / \mathrm{hour}$ ) and was haemodynamically stable. The hyperkalaemia was treated successfully with an infusion of insulin and dextrose. We discussed his medications with the pharmacist as well as the intensive care team but the cause for the hyperkalaemia remained undetermined. To further investigate the hyperkalaemia, urinary electrolytes were requested and found to show inappropriate urinary potassium retention (urinary $\mathrm{K}^{+} 10 \mathrm{mEq} / \mathrm{L}$, reference range $20-40 \mathrm{mEq} / \mathrm{L}$ ). It was this that led to the diagnosis of trimethoprim induced hyperkalaemia. By day 6 of co-trimoxazole his potassium was persistently above $6 \mathrm{mmol} / \mathrm{L}$ despite medical therapy so he was commenced on continuous venovenous haemodiafiltration. On advice from the microbiology service the co-trimoxazole therapy was continued. Renal replacement therapy (RRT) was continued for 4 days with no further hyperkalaemia. 2 days after stopping RRT he developed significant hyperkalaemia again. In order to counter the potassium-retaining effect of trimethoprim the patient was treated with furosemide intravenous boluses $(50 \mathrm{mg})$ and a normal saline infusion to promote kaliuresis. This resulted in a prompt return of serum potassium to the normal range without requiring the re-initiation of RRT or switching the anti-microbial regimen to a less effective agent.

Unfortunately despite aggressive therapy the patient's respiratory function deteriorated and following discussion

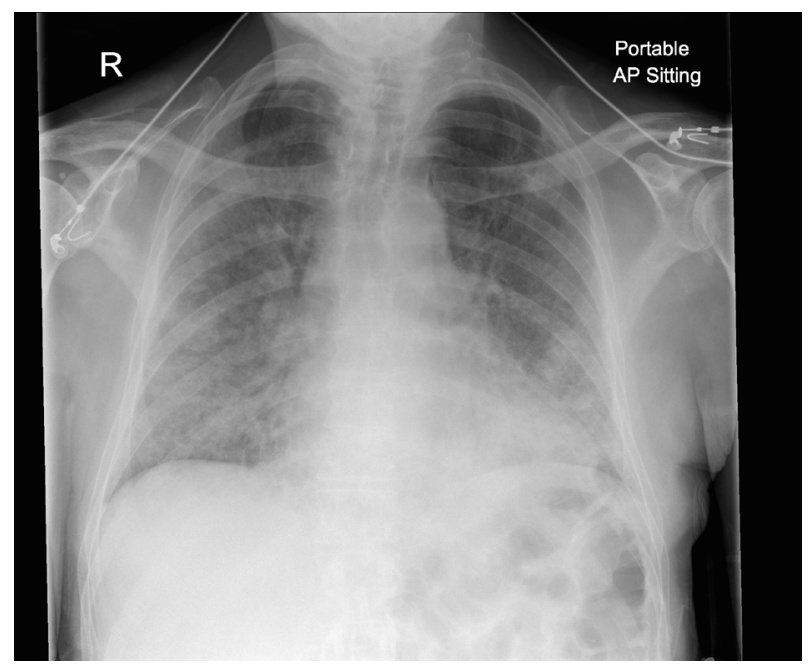

Figure 1. Chest X-ray on second admission. 


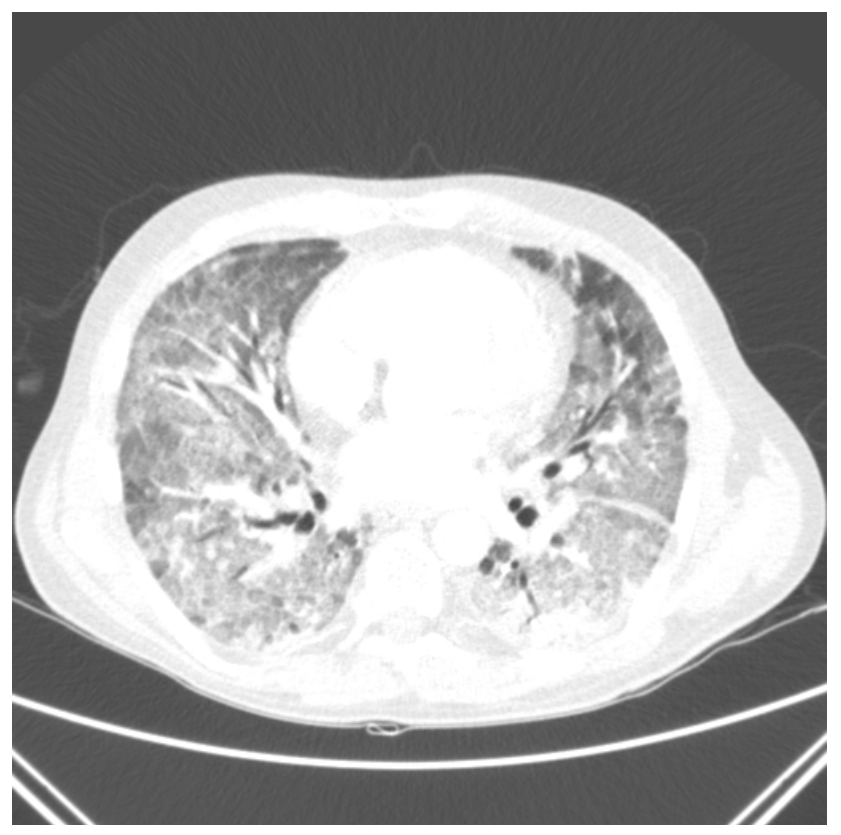

Figure 2. CT scan of the thorax.

with his family it was agreed to withdraw therapy and he succumbed to the illness.

We believe that this is the first published case of using these conservative methods based on understanding the mechanism of action of trimethoprim, to manage trimethoprim-induced hyperkalaemia.

\section{Discussion}

Co-trimoxazole is a combination antibiotic made up of trimethoprim and sulphamethoxazole that is first line treatment for both mild and severe PJP [1]. Despite its documentation in the literature since 1983 [2], the complication of hyperkalaemia secondary to co-trimoxazole seems to be poorly recognised by physicians. It wasn't until 1993 that the renal retention of potassium secondary to the trimethorpim component was demonstrated and further studies on the mechanism of hyperkalaemia were done and the real incidence of this complication was discovered [3]. In 1997 a study by Safrin et al. comparing three PJP regimes found that hyperkalaemia $\left(\mathrm{K}^{+}>5.6\right.$ $\mathrm{mmol} / \mathrm{L}$ ) occurred in $20.3 \%$ of patients treated with co-trimoxazole, $10.3 \%$ treated with dapsone and trimethoprim and only 3.5\% treated with clindamycin and primaquine [4]. A further study looking at patients taking low dose oral co-trimoxazole as outpatients demonstrated that, while only a small proportion of patients developed significant hyperkalaemia (6\%), almost all patients (81.5\%) had an increase in their potassium levels from baseline, while other studies suggest that between $76 \%$ and $100 \%$ of patients see an increase [3] [5]. This would seem to suggest that trimethoprim has a generalised effect on potassium homeostasis but that some are more susceptible to its effects leading to clinically significant hyperkalaemia. Those who are more likely to develop significant hyperkalaemia are those with renal impairment, those receiving high doses of trimethoprim, those on other drugs that may affect potassium homeostasis (NSAIDs, potassium sparing diuretics, angiotensin-converting enzyme (ACE) inhibitors), older patients, those with hypoaldosteronism or those with HIV infection [3] [6]. AIDS itself has been independently shown to cause hyperkalaemia by causing subclinical or overt hypoadrenalism, hyporeninaemic hypoaldosteronism and renal impairment and was found in $16 \%$ of hospitalised patients with AIDS [6]. This, and the longer duration of co-trimoxazole required in patients with HIV, at least partly explains that severe hyperkalaemia is less often seen in non-HIV infected patients [7].

The mechanism of hyperkalaemia seen in patients taking co-trimoxazole is trimethoprim's similar structure and function to amiloride, the potassium sparing diuretic. It reversibly inhibits the sodium channels in the distal nephron causing reduced uptake of sodium resulting in a decreased electrochemical gradient to drive potassium excretion (Figure 3). This results in increased sodium excretion and reduced potassium excretion by around $40 \%$ [3] [5] [7] [8]. This effect appears to be dose dependent. Trimethorpim is 50\% - 60\% renally excreted and reaches 
high concentrations in the kidney within 24 hours. Even low doses of trimethoprim, $200 \mathrm{mg}$ for example, produces high enough concentrations in the kidney to have this effect. The onset of hyperkalaemia occurs between $3-10$ days with a mean of 4 - 5 and a peak level at 7 - 10 days [3] [9]. Another renal effect that trimethoprim can have is to decrease creatinine clearance, falsely giving the impression of renal impairment, which may hide the potassium sparing mechanism as the cause of the hyperkalaemia [3]. As long as the patient has a good urine output, normal acid base balance and the creatinine rise is not large then the potassium sparing mechanism is more likely to be responsible for the rising potassium level.

Co-trimoxazole is the first line therapy for PJP but non-response at 8 days or side effects would usually lead to a change in therapy. Unfortunately there is little evidence on which to base our treatment decisions as all the major trials for severe disease were done in the pre-steroid era. In this case the patient was not immunosuppressed with HIV but with immunosuppressive drugs and there is again very little data for these patients available [1] [10]. It was felt by our microbiology consultants that if we could manage his hyperkalaemia then we should continue the co-trimoxazole.

We managed his hyperkalaemia by causing kaliuresis with saline and furosemide to counter the potassium retaining effects of the trimethorpim. The saline ensured adequate circulating volume and good renal perfusion. The furosemide acts on the sodium, potassium and chloride co-transporter in the luminal membrane of the ascending loop of Henle (Figure 4). It causes increased potassium excretion as well as increased sodium and water excretion. Both the saline and furosemide cause increased urinary flow rates helping to remove the drug from the kidney as well as increasing sodium delivery to the distal tubule, which has been shown in animal models, to nullify the potassium retaining effects of trimethoprim [3]. We believe that this is the first publication where this theory has been successfully used to manage hyperkalaemia in a real patient and allowed us to avoid restarting renal replacement therapy.

Distal nephron

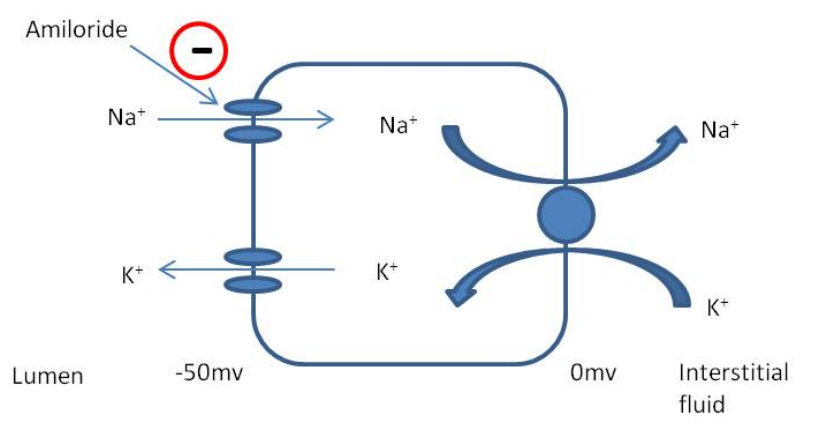

Figure 3. The mechanism of action of amiloride.

Ascending loop of Henle

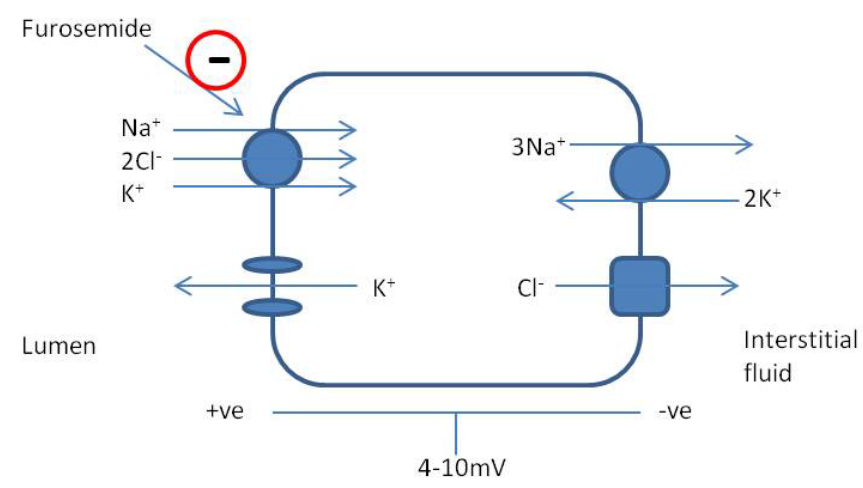

Figure 4. The mechanism of action of furosemide. 


\section{Conclusions}

In summary, we describe a case of trimethoprim induced hyperkalaemia that was successfully managed by using saline and furosemide to reverse the renal retention of potassium, thus allowing the drug to be continued without invasive treatments such as haemodiafiltration.

Learning points:

1) Co-trimoxazole (trimethorpim) can cause hyperkalaemia via a potassium-sparing diuretic effect;

2) This occurs from day 3 onwards;

3) Nearly all patients will see a mild rise in potassium levels;

4) Risk factors for trimethoprim induced hyperkalaemia include increasing dose, renal failure, HIV, other drugs affecting potassium homeostasis and age;

5) The hyperkalaemia can be managed by conservative measures using furosemide and saline to promote kaliuresis.

\section{Acknowledgements}

Consent for publication was approved by the partner of the deceased patient. The authors declare that they have no conflict of interests.

\section{References}

[1] Sax, P.E. (2012) Treatment of Pneumocystis Infection in HIV-Infected Patients. Uptodate Article. http://www.uptodate.com/contents/treatment-of-pneumocystis-infection-in-hiv-infected-patients

[2] Kaufman, A.M., Hellman, G. and Abramson, R.G. (1983) Renal Salt Wasting and Metabolic Acidosis with Trimethoprim-Sulfamethoxazole Therapy. The Mount Sinai Journal of Medicine, 50, 238-239.

[3] Perazella, M.A. (2000) Trimethoprim-Induced Hyperkalaemia: Clinical Data, Mechanism, Prevention and Management. Drug Safety, 22, 227-236. http://dx.doi.org/10.2165/00002018-200022030-00006

[4] Safrin, S. and Finkelstein, D.M. (1997) Comparison of Oral Agents for the Treatment of Pneumocystis carinii Pneumonia [Letter]. Annals of Internal Medicine, 126, 407-408 http://dx.doi.org/10.7326/0003-4819-126-5-199703010-00012

[5] Alappan, R., Buller, G.K. and Perazella, M.A. (1999) Trimethoprim-Sulfamethoxazole Therapy in Outpatients: Is Hyperkalemia a Significant Problem? American Journal of Nephrology, 19, 389-394. http://dx.doi.org/10.1159/000013483

[6] Velazquez, H., Perazella, M.A., Wright, F.S. and Ellison, D.H. (1993) Renal Mechanism of Trimethoprim-Induced Hyperkalemia. Annals of Internal Medicine, 119, 296-301. http://dx.doi.org/10.7326/0003-4819-119-4-199308150-00008

[7] Stangoe, D., Jenkins, N. and Coakes, J. (2013) A Case or Refractory Hyperkalaemia in an HIV-Positive Patient with Pneumoncystis jirovecii Pneumonia. JCIS, 14, 259-262.

[8] Antoniou, T., Gomes, T., Mamdani, M.M., Yao, Z., Hellings, C., Garg, A.X., Weir, M.A. and Juurlink, D.N. (2011) Trimethoprim-Sulfamethoxazole Induced Hyperkalaemia in Elderly Patients Receiving Spironolactone: Nested CaseControl Study. BMJ, 343, Article ID: d5228. http://dx.doi.org/10.1136/bmj.d5228

[9] Greenberg, S., Reiser, I.W., Chou, S.Y. and Porush, J.G. (1993) Trimethoprim-Sulfamethoxazole Induces Reversible Hyperkalemia. Annals of Internal Medicine, 119, 291-295. http://dx.doi.org/10.7326/0003-4819-119-4-199308150-00007

[10] Bennett, N.J. (2013) Pneumocystis jirovecii Pneumonia. Emedicine Article. http://emedicine.medscape.com/article/225976-overview 
Scientific Research Publishing (SCIRP) is one of the largest Open Access journal publishers. It is currently publishing more than 200 open access, online, peer-reviewed journals covering a wide range of academic disciplines. SCIRP serves the worldwide academic communities and contributes to the progress and application of science with its publication.

Other selected journals from SCIRP are listed as below. Submit your manuscript to us via either submit@scirp.org or Online Submission Portal.
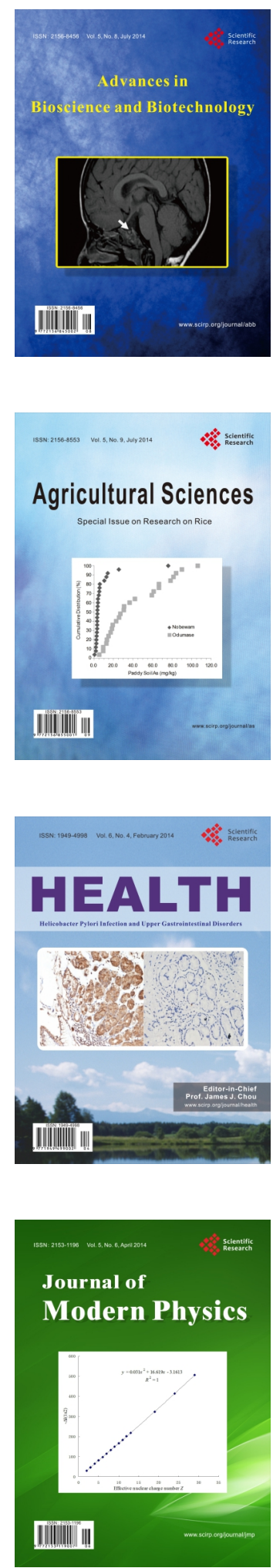
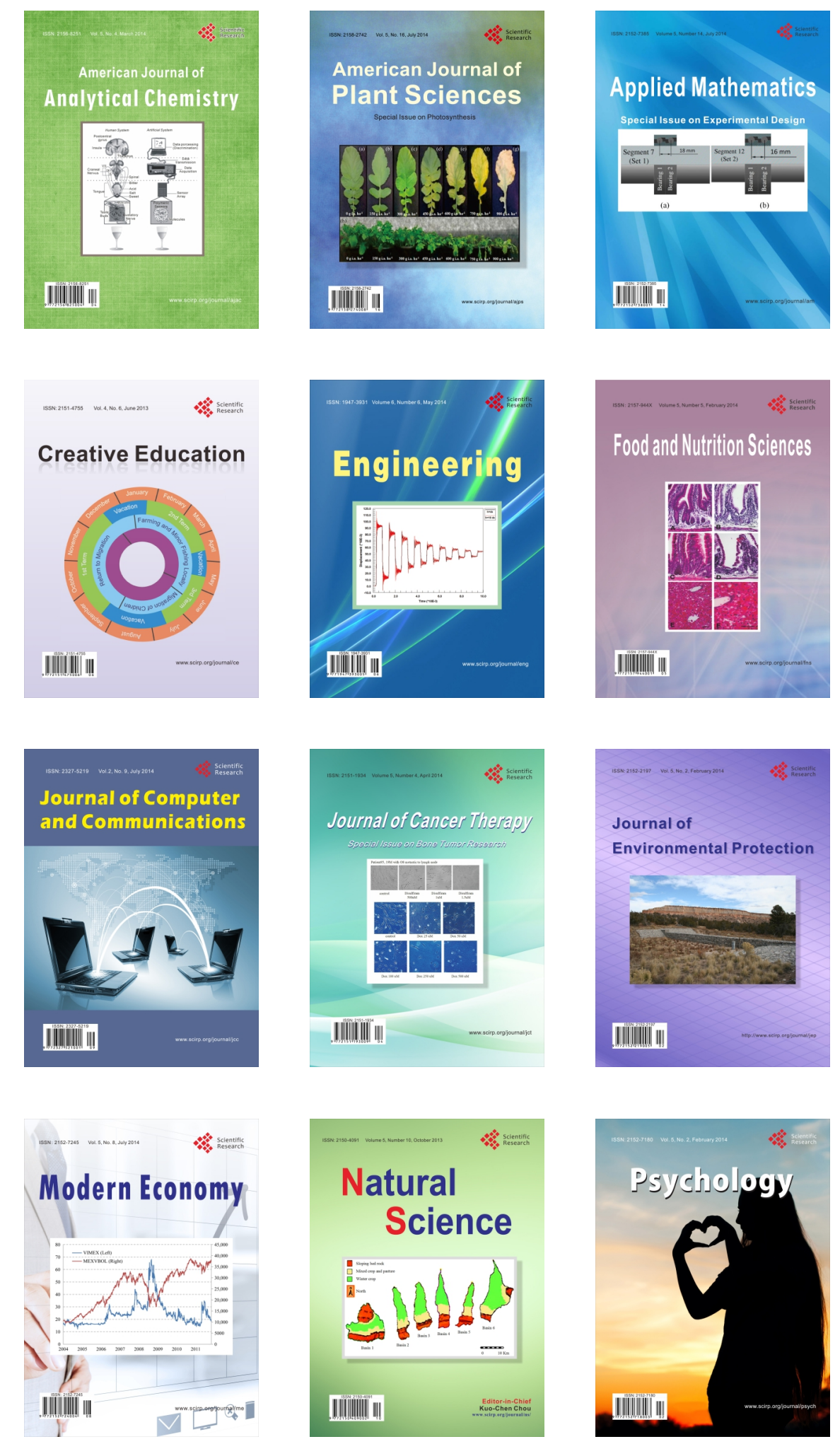